\title{
Estado nutricional de pacientes críticos com úlcera por pressão em terapia enteral internados em UTI
}

\author{
Nutritional status of critically ill patients with pressure ulcers \\ undergoing enteral therapy admitted to the ICU
}

\author{
Thamires Stephanie Alves Batista ${ }^{1 *}$, Ramara Kadija Fonseca Santos², Barbara Rafaela Santos \\ da Rocha ${ }^{1}$, Analícia Rocha Santos Freire ${ }^{1}$, Liliane Viana Pires ${ }^{1,3 *}$.
}

\begin{abstract}
RESUMO
O estudo avaliou a relação entre o estado nutricional e a evolução clínica de úlcera por pressão (UPP) em pacientes em uso de Terapia Nutricional Enteral (TNE) internados em Unidade de Terapia Intensiva (UTI). Para isso, um estudo observacional foi realizado com 26 pacientes com UPP internados em hospital público. O estado nutricional (antropometria, biomarcadores e dieta) foi avaliado na admissão do paciente na pesquisa (T0) e após uma semana de acompanhamento (T1). A media de idade foi de 54,6 anos e 58\% foi do sexo masculino, sendo que $47 \%$ e $33 \%$ dos adultos apresentaram sobrepeso e desnutrição, respectivamente. Enquanto 56\% dos idosos apresentaram desnutrição no T0 e 33\% no T1. Foi observada redução nos valores médios de albumina sérica no T1. Considerando o estágio prévio da UPP, observou-se que $54 \%$ dos pacientes mantiveram-se estáveis no T1, enquanto $42 \%$ apresentaram piora e houve melhora em apenas um paciente. A dieta ofertada era hiperproteica e sua composição não diferiu nos dois tempos. Observou-se que $61 \%$ dos pacientes não atingiram as necessidades energéticas no T0 e $58 \%$ no T1. Assim, não foram observadas melhoras nos parâmetros bioquímicos e cicatrização dos pacientes com UPP internados em UTI em TNE.
\end{abstract}

Palavras-chave: Úlcera por pressão; Estado nutricional; Unidade de Terapia Intensiva; Terapia Nutricional; Nutrição.

\section{ABSTRACT}

The study evaluated the relationship between nutritional status and the clinical evolution of pressure ulcers (PU) in patients using Enteral Nutritional Therapy (ENT) admitted to an Intensive Care Unit (ICU). For this purpose, an observational study was conducted with 26 patients with PU admitted to a public hospital. Nutritional status (anthropometry, biomarkers, and diet) was assessed upon patient admission to the research (T0) and after one week of follow-up (T1). The mean age was 54.6 years and 58\% were male, $47 \%$ and $33 \%$ of the adults were overweight and malnourished, respectively. While $56 \%$ of the elderly presented malnutrition at $\mathrm{T} 0$ and $33 \%$ at $\mathrm{T} 1$. Reduction in mean serum albumin values was observed at $\mathrm{T} 1$. Considering the previous stage of the PU, it was observed that $54 \%$ of patients remained stable at $\mathrm{T} 1$, while $42 \%$ worsened and only one patient improved. The diet offered was hyperprotein and its composition did not differ between the two times. It was observed that $61 \%$ of the patients did not meet their energy

\footnotetext{
${ }^{1}$ Departamento de Nutrição, Centro de Ciências Biológicas e da Saúde, Universidade Federal de Sergipe.

${ }^{2}$ Programa de Pós-graduação em Ciências da Saúde, Centro de Ciências Biológicas e da Saúde,

Universidade Federal de Sergipe.

${ }^{3}$ Programa de Pós-graduação em Ciências da Nutrição, Centro de Ciências Biológicas e da Saúde,

Universidade Federal de Sergipe. *E-mail: 1vianapires@gmail.com
} 
requirements at $\mathrm{T} 0$ and $58 \%$ at $\mathrm{T} 1$. Thus, no improvements were observed in biochemical parameters and healing of patients with PU admitted to the ICU on ENT.

Keywords: Pressure ulcer; Nutritional status; Intensive care unit; Nutritional Therapy; Nutrition.

\section{INTRODUÇÃO}

Úlceras por pressão (UPP) são lesões localizadas na pele e/ou no tecido ou estrutura subjacente, oriundas de pressão isolada que causa isquemia e necrose do tecido ou de pressão combinada com fricção e/ou cisalhamento, normalmente em uma proeminência óssea, que pode afetar os leitos capilares subjacentes, contribuindo para a hipóxia local (MERVIS; PHILLIPS, 2018). A desnutrição no contexto de distúrbios que incluem a desnutrição energético-proteica, obesidade e concentrações anormais de micronutrientes (MUNOZ et al., 2020) está associada ao maior risco de desenvolvimento de UPP e pior cicatrização das lesões já existentes (KENNER; BATCHELORMURPHY; YAP, 2015; MOLNAR; VLAAD; GUMUS, 2016).

Em situação de desnutrição há redução na produção de fibroblastos, na neoangiogênese e na síntese de colágeno, dificultando a cicatrização das UPP (MOLNAR; VLAAD; GUMUS, 2016). Além disso, a desnutrição aumenta a suscetibilidade do indivíduo à infecção, o que contribui para maior incidência de complicações e maior tempo de internação nas Unidades de Terapia Intensiva (UTI) (MOLNAR; VLAAD; GUMUS, 2016; TAYYIB; COYER, 2016). Ademais, nutrientes específicos contribuem para o processo de cicatrização (QUAIN; KHARDORI, 2015; STECHMILLER, 2010), dentre eles, a vitamina A, C e E, e os minerais zinco, cálcio, magnésio e ferro, cuja a deficiência reduz a síntese de colágeno e elastina e dificulta o processo de cicatrização (KOGAN; SOOD; GARNICK, 2017; MOORES, 2013; QUAIN; KHARDORI, 2015).

Dessa forma, a nutrição adequada é de suma importância para a recuperação do paciente tanto em relação à doença de base quanto para redução do risco de desenvolvimento de UPP e melhor cicatrização das mesmas (SBNEP, 2018). No intuito de verificar a hipótese que o melhor estado nutricional proporcionado pela Terapia Nutricional Enteral (TNE) está associado à redução na incidência das UPP e melhor cicatrização daquelas já existentes em pacientes críticos, este estudo objetiva avaliar o estado nutricional de indivíduos com UPP em TNE internados em UTI. 


\section{MÉTODOS}

\section{Delineamento do estudo}

Trata-se de estudo observacional, desenvolvido com 26 pacientes com UPP internados na UTI de um hospital público de Sergipe. A amostra foi inicialmente constituída de 40 pacientes, destes sete pacientes apresentavam impossibilidade de serem reavaliados, três pacientes receberam alta da UTI; dois foram a óbito; um iniciou dieta via oral e um não foi autorizado pelo responsável a continuar na pesquisa, assim, 26 pacientes permaneceram no estudo.

As informações acerca da presença de UPP, estado nutricional, prescrição dietoterápica e dos resultados de exames laboratoriais foram obtidas no prontuário médico no momento de admissão do paciente na pesquisa (T0) e após uma semana (T1).

\section{Critérios de inclusão}

Ter idade acima de 18 anos, apresentar UPP, estar em nutrição enteral exclusiva, não apresentar queimaduras de ampla extensão e estar internado por no mínimo 48 horas.

\section{Critérios de exclusão}

Não permanecer internado por pelo menos uma semana na UTI.

\section{Aspectos éticos}

Este estudo foi conduzido de acordo com as diretrizes estabelecidas pela Declaração de Helsinki e todos os procedimentos envolvendo os pacientes avaliados foram aprovados pelo Comitê de Ética em Pesquisa da Universidade Federal de Sergipe sob o parecer número 829.061. O consentimento escrito livre e esclarecido foi obtido de todos os responsáveis pelos participantes incluídos no estudo.

\section{Avaliação nutricional}

Avaliação antropométrica foi realizada na admissão dos pacientes no estudo. A estatura (cm) foi estimada utilizando a fórmula de Chumlea, Guo, Steinbaugh (1994) a partir dos valores da altura do joelho $(\mathrm{cm})$. O peso $(\mathrm{kg})$ foi estimado por meio da fórmula de Chumlea et al. (1988) e calculado o Índice de Massa corpórea (IMC). Valores da circunferência do braço $(\mathrm{CB})(\mathrm{cm})$ e da circunferência da panturrilha $(\mathrm{CP})(\mathrm{cm})$ também foram obtidos.

O estado nutricional segundo o IMC foi classificado conforme os pontos de corte estabelecidos pela OMS (1998) para adultos e por Lipschitz (1994) para idosos. Os 
valores da CB e CP foram classificados de acordo Blackburn \& Thornton (1979) e OMS (1995), respectivamente.

A avaliação dietética foi determinada pela informação contida em prontuário quanto ao volume diário de dieta infundida. Esses valores foram registrados e reavaliados uma semana após a admissão no estudo. As necessidades nutricionais foram calculadas pela fórmula de Harris-Benedict (1984), utilizando o fator injúria correspondente a cada doença de base e o peso estimado no momento da admissão do paciente no estudo.

Os resultados dos exames bioquímicos (hematócrito, hemoglobina, leucócitos, linfócitos, albumina sérica, ureia e creatinina) também foram obtidos nos prontuários correspondentes a cada tempo de avaliação (T0 e T1).

\section{Avaliação estatística}

Foi realizada análise descritiva dos dados e os resultados apresentados em frequência absoluta e relativa para dados categóricos e média e desvio padrão para dados contínuos. O teste de Shapiro-Wilk foi aplicado no intuito de avaliar a normalidade na distribuição dos dados. Para amostras com distribuição normal foram aplicados os testes t-Student para amostras dependentes ou o teste pareado de Wilcoxon. Foram consideradas diferenças significativas $\mathrm{p}$-valor $<0,05$.

\section{RESULTADOS}

Dos 26 pacientes avaliados, verificou-se que a maioria era do sexo masculino (58\%). A média de idade foi de 54,6 $\pm 17,1$ anos, sendo $17(65,4 \%)$ pacientes adultos e 9 $(34,6 \%)$ idosos.

Os diagnósticos clínicos de maior prevalência nos pacientes avaliados foram relacionados às doenças ou complicações neurológicas e do sistema respiratório, com percentuais de $46 \%$ e $19 \%$, respectivamente.

Os resultados da avaliação antropométrica estão apresentados na tabela 1. Não foram observadas diferenças significativas nos parâmetros antropométricos ao final do estudo. A avaliação do IMC mostrou predomínio de pacientes com sobrepeso tanto em adultos (47\%) quanto em idosos (56\%). A prevalência de desnutrição nos idosos foi de $10 \%$ e nos adultos $33 \%$. Os valores da CB mostraram que a maior parte dos pacientes foram classificados como eutróficos. Entretanto, ao avaliar a medida da CP nos idosos observou-se que no T0 a maioria (56\%) apresentou desnutrição, sendo este valor reduzido a $33 \%$ no T1. 
Tabela 1 - Caracterização antropométrica dos pacientes com UPP internados em UTI.

\begin{tabular}{lccc}
\hline Variáveis & T0 & T1 & $p$-valor** \\
\hline Peso estimado $(\mathrm{kg})$ & $62,10 \pm 14,37$ & $62,58 \pm 12,72$ & 0,742 \\
Estatura estimada $(\mathrm{m})$ & $1,61 \pm 0,08$ & $1,61 \pm 0,08$ & - \\
Circunferência do braço $(\mathrm{cm})^{\text {Aircunferência da Panturrilha }(\mathrm{cm}) *}$ & $28,46 \pm 4,21$ & $28,91 \pm 4,12$ & 0,367 \\
${\text { IMC estimado }\left(\mathrm{kg} / \mathrm{m}^{2}\right)}^{\text {Adulto }}{ }^{(\mathrm{n}=17)}$ & $32,06 \pm 3,48$ & $31,42 \pm 3,61$ & 0,412 \\
$\quad$ & & & \\
$\quad$ Idoso $^{(\mathrm{n}=09)}$ & $22,48 \pm 5,49$ & $23,22 \pm 5,53$ & 0,336 \\
\hline
\end{tabular}

Dados apresentados em média \pm desvio-padrão referentes ao momento da admissão na pesquisa (T0) e uma semana após (T1). *Apenas para os pacientes idosos. Abreviaturas: UPP: Úlcera por Pressão; UTI: Unidade de Terapia Intensiva; IMC: Índice de Massa Corporal. Valores de normalidade do IMC: Adultos: 18,5 a $24,9 \mathrm{~kg} / \mathrm{m}^{2}$ (OMS, 1998); Idosos: 22 a $27 \mathrm{~kg} / \mathrm{m}^{2}$ (LIPSCHITZ, 1994). **p<0,05: diferenças significativas em relação aos tempos.

Os pacientes com UPP internados na UTI receberam dieta hiperproteica, porém, a avaliação da adequação das dietas administradas mostrou que $61 \%$ dos pacientes não atingiram as necessidades energéticas no T0 e 58\% no T1. Os valores médios de energia e proteína administradas não apresentaram diferenças significativas nos dois tempos avaliados (tabela 2).

Tabela 2 - Valores de energia e proteína, e percentual de adequação da dieta enteral administrada nos pacientes com UPP internados em UTI.

\begin{tabular}{lccc} 
Variáveis & T0 & T1 & $p$-valor* \\
\hline Energia da dieta (kcal/dia) & $1941,93 \pm 557,80$ & $1993,23 \pm 683,02$ & 0,566 \\
Proteína infundida (g/dia) & $81,55 \pm 22,53$ & $88,48 \pm 32,73$ & 0,347 \\
\% de adequação energética & $78,18 \pm 38,24$ & $80,55 \pm 42,88$ & 0,703 \\
\% de adequação proteica & $134,52 \pm 59,95$ & $144,14 \pm 76,67$ & 0,408 \\
\hline
\end{tabular}

Dados apresentados em média \pm desvio-padrão referentes ao momento da admissão na pesquisa (T0) e uma semana após (T1). Abreviaturas: UPP: Úlcera por Pressão; UTI: Unidade de Terapia Intensiva. ${ }^{*} \mathrm{p}<0,05$ : diferenças significativas em relação aos tempos.

Para compor a avaliação do estado nutricional dos pacientes com UPP, foram analisados os resultados de marcadores bioquímicos que podem indicar alterações no estado nutricional dos mesmos, conforme apresentado na tabela 3.

Tabela 3 - Marcadores bioquímicos do estado nutricional, segundo o tempo de avaliação, de pacientes com UPP internados em UTI.

\begin{tabular}{|c|c|c|c|}
\hline Variáveis & T0 & $\mathrm{T} 1$ & $p$-valor* \\
\hline Hemoglobina $(g / d L){ }^{n=26}$ & $8,90 \pm 1,87$ & $8,43 \pm 1,71$ & 0,188 \\
\hline Hematócrito (\%) ${ }^{\mathrm{n}=26}$ & $25,80 \pm 5,25$ & $24,67 \pm 4,73$ & 0,250 \\
\hline Leucócitos $\left(10^{3} / \mathrm{mm}^{3}\right)^{\mathrm{n}=26}$ & $13,98 \pm 7,65$ & $13,10 \pm 8,05$ & 0,539 \\
\hline Linfócitos $(\%)^{\mathrm{n}=25}$ & $13,24 \pm 5,88$ & $14,96 \pm 6,56$ & 0,153 \\
\hline
\end{tabular}


Creatinina sérica $(\mathrm{mg} / \mathrm{dL})^{\mathrm{n}=24}$

$$
1,01 \pm 0,90
$$

$1,01 \pm 0,83$

0,273

Ureia $(\mathrm{mg} / \mathrm{dL})^{\mathrm{n}=24}$

$62,73 \pm 35,83$

$61,38 \pm 33,30$

0,781

Albumina $(\mathrm{g} / \mathrm{L}){ }^{\mathrm{n}=09}$

$2,30 \pm 0,32$

$2,08 \pm 0,40$

$\mathbf{0 , 0 1 9}$

Dados apresentados em média \pm desvio-padrão referentes ao momento da admissão na pesquisa (T0) e uma semana após (T1). Abreviaturas: UPP: Úlcera por Pressão; UTI: Unidade de Terapia Intensiva. Valores de referência: Linfócitos 20 - 30\%; Leucócitos 4000-11000 mm Hemoglobina: 13,5 a $18 \mathrm{~g} / \mathrm{dL}$ para homens e 12 a $16 \mathrm{~g} / \mathrm{dL}$ para mulheres; Hematócrito: 40 a $54 \%$ para homens e 37 a $47 \%$ para mulheres; Creatinina sérica: 0,7 a 1,6 mg/dL para homens, 0,6 a 1,1 $\mathrm{mg} / \mathrm{dL}$ para mulheres; Ureia: 10 a $40 \mathrm{mg} / \mathrm{dL}$; Albumina: 3,5 a 5,5g/dL (CALIXTO-LIMA \& REIS, 2012). *p<0,05: diferenças significativas em relação aos tempos.

Os resultados mostraram que a maioria dos pacientes apresentou alteração nos marcadores bioquímicos (hematócrito, hemoglobina, leucócitos, linfócitos, albumina sérica, ureia e creatinina) quando comparados aos seus respectivos valores de referência, com exceção da creatinina sérica que se encontrou dentro dos valores de referência nos dois momentos da avaliação. Não foi observada melhora significativa nos marcadores bioquímicos no T1. No entanto, observou-se redução nos valores de albumina no T1 ( $p=0,019)$.

Ao classificar as UPP de acordo com o estágio, verificou-se que o estágio III foi o mais prevalente (46\%), seguido dos estágios IV (23\%), II (19\%) e I (12\%). A presença isolada da UPP na região sacral foi de $85 \%$. No T1, observou-se que $42 \%(\mathrm{n}=11)$ dos pacientes tiveram piora da UPP, 54\% ( $\mathrm{n}=14)$ mantiveram a UPP estável em relação ao diagnóstico em T0 e apenas um paciente melhorou a UPP do estágio IV para o III. Todos os pacientes que apresentavam UPP em estágio I evoluíram para o II (totalizando 27\% indivíduos com UPP no estágio II), um paciente que estava em estágio II em T0 piorou e teve a UPP classificada em estágio III (totalizando $27 \%$ no estágio III) e sete pacientes evoluíram do estágio III para o IV (totalizando 46\% no estágio IV).

\section{DISCUSSÃO}

Este estudo mostrou que o tratamento dos pacientes em UTI, considerando a TNE, o estado nutricional e a idade, não foi suficiente para promover melhoras nos parâmetros bioquímicos e cicatrização das UPP. Importante destacar que, no presente estudo, o percentual de inadequação na administração da dieta mostrou-se elevado, aumentando o risco de déficit nutricional e de piora da cicatrização, apresentando-se semelhante aos achados em estudos desenvolvidos a nível mundial com $60 \%$ da adequação energética e 53\% no teor de proteína administrado (CHAPPLE et al., 2016; WEIJS et al., 2014).

A terapia nutricional individualizada iniciada precocemente reduz o risco de complicações associadas às UPP (MUNOZ et al., 2020). De acordo com as diretrizes 
nutricionais da American Society for Parenteral e Enteral Nutrition (ASPEN) e a Society for Critical Care Medicine, a alimentação do paciente em cuidado intensivo deve ser iniciada entre as 24 e 48 horas após admissão na UTI e a dieta hiperproteica apresenta-se benéfica na redução da mortalidade dos pacientes em UTI (MEHTA et al., 2017). Ademais, a via de administração e a formulação da dieta deve ser precisamente avaliada considerando capacidade gastrointestinal, tolerância, medicamentos e gravidade da doença (MUNOZ et al., 2020). Ainda, a suplementação nutricional parece ser uma maneira eficiente de repor deficiências de macro e micronutrientes no organismo e de fornecer outros nutrientes para preservar o tecido cutâneo, fortalecer a resistência tecidual e promover a reparação dos tecidos (SBNPE; ASBRAN; SBCM, 2011).

A prevalência de UPP em idosos é explicada pelo envelhecimento da pele, presença de doenças crônico-degenerativas e redução do função imune (MERVIS; PHILLIPS, 2018). Porém, além da idade outros fatores como a doença de base, tempo de internação, terapia medicamentosa, estado nutricional prévio e comorbidades associadas podem aumentar o risco do desenvolvimento de UPP (CLARK; YOUNG; FALLOW, 2018; DEMARRÉ et al., 2015), o que faz da sua gênese um fenômeno multifatorial (COX; RASMUSSEN, 2014) e justifica a presença de UPP em indivíduos mais jovens internados em UTI (SILVA et al., 2017).

Neste estudo, as doenças neurológicas compuseram $46 \%$ dos diagnósticos clínicos dos pacientes analisados, como observado em outros estudos, nos quais as doenças neurológicas foram as principais causas de hospitalização seguidas das neoplasias, doenças cardiocirculatórias e do sistema urinário (BLANES et al., 2004; COSTA et al., 2015). Estudo recente mostrou que 82,6\% das UPP surgiram em indivíduos com tempo de internação maior que 10 dias (COSTA et al., 2015). As doenças neurológicas contribuem para o aumento do tempo de internação, fator de risco para UPP em pacientes internados em UTI (MERVIS; PHILLIPS, 2018).

A avaliação dos marcadores antropométricos é utilizada para estabelecer os riscos de UPP em pacientes internados e deve ser realizada de maneira precisa (MUNOZ et al., 2020). No presente estudo, a avaliação do estado nutricional a partir da classificação do IMC mostrou a presença de indivíduos com desnutrição, eutrofia e sobrepeso/obesidade. Porém, o estado nutricional eutrófico observado mediante análise da CB sugere que não houve comprometimento da massa muscular dos pacientes quando avaliados por esse parâmetro. Em contrapartida, apesar da baixa prevalência de idosos desnutridos conforme 
o IMC e os valores da CB mostrarem eutrofia nessa população, a avaliação pela medida da CP nos idosos, a qual é considerada mais sensível para aferir a massa muscular do que a circunferência do braço, de acordo com OMS (1995), mostrou elevado percentual de idosos desnutridos.

A desnutrição contribui para o aumento na incidência das UPP e piores prognósticos destas (COX; RASMUSSEN, 2014; MUNOZ et al., 2020), haja vista que, provoca atrofia muscular, redução da massa tecidual, da função imunológica e da síntese de colágeno, logo, a perda de peso involuntária de paciente crítico deve ser precisamente avaliada no intuito de prevenir o desenvolvimento de UPP (MUNOZ et al., 2020).

Por outro lado, a situação de sobrepeso/obesidade aumenta o peso descarregado no leito contribuindo para o surgimento das UPP (MEULENDIJKS et al., 2020; NESS et al., 2018). Além disso, a obesidade favorece alterações hemodinâmicas, resistência à insulina, inflamação sistêmica crônica por meio da disfunção do tecido adiposo e aumento da permeabilidade endotelial (NEULENDIJKS et al., 2020). Corroborando aos achados no presente estudo, Costa et al. (2015) não encontraram diferença significativa na incidência das UPP conforme classificação do IMC nos pacientes internados em UTI.

Observou-se também redução nos valores de albumina, bem como alterações nos valores de hemoglobina e hematócrito, sem diferença significativa entre os dois tempos avaliados. Estes achados foram corroborados por Campos et al. (2010) que observaram menores valores médios de hemoglobina e hematócrito em indivíduos com UPP quando comparados aos pacientes sem UPP. Costa et al. (2015) observaram associação significativa dos menores valores de hemoglobina e incidência de UPP em pacientes em UTI. Além disso, a hipoalbuminemia observada no T1 pode indicar depleção proteica, porém fatores não nutricionais, tais como estados de perda de proteína, função hepática, infecção aguda, fluídos corporais e inflamação podem influenciar as concentrações de albumina sérica (MUNOZ et al., 2020).

A localização da UPP também é outro fator que deve ser considerado. No presente estudo a UPP na região sacral foi a mais prevalente. Outros estudos realizados com pacientes em UTI mostraram prevalência de UPP na região sacral em percentuais variados, desde 36,4\% (RODRIGUEZ-MUNEZ et al., 2019) a 100\% (COSTA et al., 2015). Essa região é mais propensa à formação de UPP devido à grande pressão exercida pelo peso corporal durante o repouso prolongado em superfície inadequada (CAMPOS et al., 2010). 
Dentre as limitações observadas no estudo estão o intervalo de tempo entre as avaliações dos pacientes com o intuito de verificar a participação da terapia nutricional no desfecho das UPP, uma vez que apresentam etiologia complexa, além da influência das doenças de base que podem necessitar maior tempo de acompanhamento. Além disso, maior número de pacientes avaliados é necessário para que análises mais aprofundadas de fatores que podem influenciar no efeito da terapia nutricional na melhora das UPP, tais como terapia medicamentosa e história clínica do paciente, possam ser realizadas.

\section{CONCLUSÃO}

Os pacientes internados em UTI e em TNE não obtiveram melhoras quanto a cicatrização das UPP, bem como nos parâmetros bioquímicos. Ademais, o elevado percentual de inadequação na administração da dieta pode justificar os resultados encontrados, mostrando a importância do monitoramento da administração da dieta no intuito de promover adequado aporte energético-proteico e de micronutrientes importantes na prevenção e cicatrização das UPP. Ainda, aspectos como terapia medicamentosa, frequência da fisioterapia e cuidados com a pele precisam ser considerados a fim de caracterizar a população estudada e melhor explicar a presença de UPP em pacientes em UTI.

\section{Conflito de interesse}

Os autores declaram não ter nenhum conflito de interesse.

\section{AGRADECIMENTOS}

Aos pacientes da pesquisa, pela disponibilidade de participação. Ao Hospital no qual o estudo foi realizado pelo apoio e estrutura disponibilizados.

\section{REFERÊNCIAS}

BLACKBURN, G. L.; THORNTON, P. A. Nutritional assessment of the hospitalized patient. Medical Clinics of North America, v. 63, p. 11103-15, 1979.

BLANES, L.; DUARTE, I.S.; CALIL, J.Á.; FERREIRA, L.M. Avaliação clínica e epidemiológica das úlceras por pressão em pacientes internados no hospital São Paulo. Revista da Associação Médica Brasileira, v. 50, n. 2, p. 182-7, 2004.

CAMPOS, S.F.; CHAGAS, Â.C.P.; COSTA, A.B.P.; DE MELO FRANÇA, R.E.; JANSEN, A.K. Factors associated with the development of pressure ulcers: The impact of nutrition. Revista de Nutrição, v. 23, n. 5, p. 703-14, 2010. 
CHAPPLE, L.A.S.; CHAPMAN, M.J.; LANGE, K.; DEANE, A.M.; HEYLAND, D.K. Nutrition support practices in critically ill head-injured patients: A global perspective.

Critical Care, v. 20, n. 1, p. 1-11, 2016.

CHUMLEA, W. C.; GUO, S.; ROCHE, A. F.; STEINBAUGH, M. L. Prediction of body weight for the nonambulatory elderly from anthropometry. Journal of the American Dietetic Association, v. 88, n. 5, p. 564-8, 1988.

CHUMLEA, W.C.; GUOS, S.; STEINBAUGH, M.L. Prediction of stature from knee height for black and white adults and children with application to mobility-impaired or handicapped persons. Journal of the American Dietetic Association, v. 94, n. 12, p. 1385-8, 1994.

CLARK, M.; YOUNG, T.; FALLON, M. Systematic review of the use of Statistical Process Control methods to measure the success of pressure ulcer prevention.

International Wound Journal, v.15, n. 3, p. 391-401, 2018.

COSTA, A.C.O.; PINHO, C.P.S.; DOS SANTOS, A.D.A.; DO NASCIMENTO, A.C.S. Úlcera por presión: Incidencia y factores demográficos, clínicos y nutricionales asociados en pacientes de una unidad de cuidados intensivos. Nutrición Hospitalaria, v. 32, n. 5, p. 2242-52, 2015.

COX, J.; RASMUSSEN, L. Enteral nutrition in the prevention and treatment of pressure ulcers in adult critical care patients. Critical Care Nurse, v. 34, n. 6, p. 15-27, 2014.

DEMARRÉ, L.; VAN, L. A.; VAN, H.A.; VERHAEGHE, S.; GRYPDONCK, M.; LEMEY, J.; LIEVEN, A.; DIMITRI, B. The cost of prevention and treatment of pressure ulcers: A systematic review. International Journal of Nursing Studies, v. 52, n. 11, p. 1754-74, 2015.

KENNERLY, S.; BATCHELOR-MURPHY, M.; YAP, T.L. Clinical insights: Understanding the link between nutrition and pressure ulcer prevention. Geriatric Nursing, v. 36, n. 6, p. 477-81, 2015.

KOGAN, S.; SOOD, A.; GARNICK, M.S.; ZINC AND WOUND HEALING: A Review of Zinc Physiology and Clinical Applications. Wounds: A Compendium of Clinical Research and Practice, v. 29, n. 4, p. 102-6, 2017.

LIPSCHITZ, D.A. Screening for nutritional status in the elderly. Primary Care, v, 21, n. 1, p. 55-67, 1994.

MEHTA, N.M.; SKILLMAN, H.E.; IRVING, S.Y.; COSS-BU, J.A.; VERMILYEA, S.; FARRINGTON, E.A.F.; MCKEEVER, L..M.S.; HALL, A.M.; GODAY, P.S.;

BRAUNSCHWEIG, C. Guidelines for the Provision and Assessment of Nutrition Support Therapy in the Pediatric Critically Ill Patient. Pediatric Critical Care Medicine, v. 18, n. 7, p. 675-715, 2017.

MERVIS, J.S.; PHILLIPS, T.J. Pressure ulcers: Pathophysiology, epidemiology, risk factors, and presentation. Journal of the American Academy of Dermatology, v. 81, n. 4, p. 881-90, 2019.

MEULENDIJKS, A.M.; FRANSSEN, W.M.A.; SCHOONHOVEN, L.; NEUMANN, H. A scoping review on Chronic Venous Disease and the development of a Venous Leg Ulcer: The role of obesity and mobility. Journal of Tissue Viability, v. 29, n. 3, p. 190$6,2020$. 
MOLNAR, J.A.; VLAD, L.G.; GUMUS, T. Nutrition and chronic wounds: Improving clinical outcomes. Plastic and Reconstructive Surgery, v. 138, n. 3, p. 71S-81S, 2016.

MOORES, J. Vitamin C: a wound healing perspective. British Journal of Community Nursing,v. 18 (Supl.12) p. S6-11, 2013.

MUNOZ, N.; POSTHAUER, M.E.; CEREDA, E.; SCHOLS, J.M.G.A.; HAESLER, E. The Role of Nutrition for Pressure Injury Prevention and Healing: The 2019 International Clinical Practice Guideline Recommendations. Advances in Skin \& Wound Care, v. 33, n. 3, p. 123-36, 2020.

NESS. S.J.; HICKLING, D.F.; BELL, J.J.; COLLINS, P.F. The pressures of obesity: The relationship between obesity, malnutrition and pressure injuries in hospital inpatients. Clinical Nutrition, v. 37, n. 5, p. 1569-74, 2018.

QUAIN, A.M.; KHARDORI, N.M. Nutrition in Wound Care Management: A Comprehensive Overview. Wounds, v. 27, n. 12, p. 327-35, 2015.

RODRÍGUEZ-NÚÑEZ, C.; IGLESIAS-RODRÍGUEZ, A.; IRIGOIEN-AGUIRRE, J.; GARCÍA-CORRES, M.; MARTÍN-MARTÍNEZ, M.; GARRIDO-GARCÍA, R. Nursing records, prevention measures and incidence of pressure ulcers in an Intensive Care Unit. Enfermería Intensiva, v. 30, n. 3, p. 135-43, 2019.

SBNPE; ABRAN; SBCM. Sociedade Brasileira de Nutrição Parenteral e Enteral; Associação Brasileira de Nutrologia; Sociedade Brasileira de Clínica Médica. Terapia Nutricional para portadores de úlceras por pressão. Proj Diretrizes. 2011. Disponível em:

https://amb.org.br/files/_BibliotecaAntiga/terapia_nutricional_para_pacientes_portadore s_de_ulceras_por_pressao.pdf Acesso em: 31 de agosto de 2021.

SILVA, D.R.A.; BEZERRA, S.M.G.; COSTA, J.P.; LUZ, M.H.B.A.; LOPES, V.C.A.; NOGUEIRA, L.T. Pressure ulcer dressings in critical patients: A cost analysis. Revista da Escola de Enfermagem da USP, v. 51, p. 1-7, 2017.

SOCIEDADE BRASILEIRA DE NUTRIÇÃO PARENTERAL E ENTERAL (SBNPE). Diretrizes Brasileiras de Terapia Nutricional no Paciente Grave. Braspen Journal, v. 33 (supl 1), p. 2-36, 2018.

STECHMILLER, J.K. Understanding the Role of Nutrition and Wound Healing. Nutrition in Clinical Practice, v. 25, n. 1, p. 61-8, 2010.

TAYYIB, N.; COYER, F. Effectiveness of pressure ulcer prevention strategies for adult patients in intensive care units: a systematic review protocol. JBI database of systematic reviews and implementation reports, v. 14, n. 3, p. 35-44, 2016.

WEIJS, P.J.M.; LOOIJAARD, W.G.P.M.; BEISHUIZEN, A.; GIRBES, A.R.J.; OUDEMANS-VAN, S.H.M. Early high protein intake is associated with low mortality and energy overfeeding with high mortality in non-septic mechanically ventilated critically ill patients. Critical Care, v. 18, n. 6, p. 701, 2014.

World Health Organization (WHO). Obesity: preventing and managing the global epidemic. Report of a WHO Consulation. Genebra. 1998. 
Recebido em: 01/06/2021

Aprovado em: 20/06/2021

Publicado em: 30/06/2021 